\title{
Schedule Planning Proceeding of Transfer Batches in Flexible Automated Lines While Sets are Forming and Limits are Recognized
}

\author{
K.V. Krotov ${ }^{1, ~}{ }^{*}, A . Y$. Drozin $^{1}$, and E.N. Zaikina ${ }^{1}$ \\ ${ }^{1}$ Sevastopol State University, Sevastopol, Russia
}

\begin{abstract}
The hierarchical approach while solving the problem of complex scheduling of transferred batches of units using flexible transfer lines is considered, when sets and time limits of flexible transfer lines are formed. Mathematical model of hierarchical game to optimize a mixture of transferred batches is developed. The groups of sets are processing during time intervals of definite lengths. The schedules of batches of units are optimized, using the mathematical model. The way of optimizing a mixture of groups of sets while resulting batches are formed, is developed.
\end{abstract}

The output in high-mixed productions recognizes the processing of transferred batches (TB) using Flexible Automated Lines (FAL). The FALs are made as a sequence of flexible technological places (FTP). Each FTP can be rearranged to process different type of units. The increasing of effectiveness is associated with a scheduling planning problem. The result of such problem solving is a project schedule of FAL activity. The input of this task is an industrial program formed by Enterprise's Automatic Control System (ASC). The ASC gets a set of items to be processed if FAL. The unified items (units) are the units with the same processing time intervals. The time intervals to rearrange the FTP are equal. The time intervals, used to rearrange the FTP to proceed these types of items are equal, too. While time intervals of processing and rearranging FTP are inhomogeneous enough, the effectiveness of scheduling depends on the mixture of transferred batches. In such case the planning of TB assumes the set of batch and the schedule of processing using FAL is considered [1]. The problem of complex processing schedule of TS using FAL while the sets of items are formed and the time limits of FAL acting is developed. The set if items is a set of different kinds of items which are processed in FAL. The number of items of each kind which are included in a set, is preset. The ASC program gets a number of each kind of sets and the time intervals to process a set of batches using FAL.

Hierarchical approach is used to solve a problem [1]. It considers to decompose the generalized function in decision support system of FAL. The function if decomposed to a set of sub-functions, which are hierarchically ordered. The problem solving is implemented using the hierarchical levels:

- the first (upper) level - defines of solutions of structure TB;

*Corresponding author: krotov_k1@mail.ru 
- the second level - defines the solutions of groups of TB, which are proceed during the given time intervals;

- the third level - defines the schedule of TB processing, using FAL for solutions of groups of TB is formed on the second level.

To solve a problem of scheduling while sets are proceed and batches are formed, using time interval limits, some subtasks need to be made. The subtasks are: to define of structure TB; to define of structure of groups of TBs, which are proceed, using given time intervals; to define schedules of TBs processing using FAL; to allocate the items, proceed using FAL during given time interval to sets.

The modern ways of complex schedules of processing of batches in multi-stage systems (FAL, in particular case), are realizing the scheduling of sets of materials, which are proceeding in non-stop (continuous) productions [2,3]. The ways can define the order of definite batches implementations [4,5] and realize the decisions of a party set and the order of operations using one or several parallel devices [6,7]. The review of articles defines there are no ways to form a structures of TBs in multi-stage systems, when problem order is unlimited. There are no ways to determine a structures of TBs while the sets are formed. There are no ways to solve a scheduling problem, when the time intervals of FAL activity are limited. The purpose of the research is to improve the ways of complex scheduling of TBs proceeding in FAL. The hierarchical game model is developed to solve the problem. Using such a game defines the structures of batches, the structures of groups of batches and the schedule of processing them using FAL; the initial solve of structures of TB and an optimized groups of TB is defined too. The scheduling is realized by three-levels formed solving. The first-level gamer is forming the structures of TB. The second-level gamer is forming the groups of TBs, the third-level gamer is forming the scheduling of groups of TBs processing. The order of the players' moves is:

1) the first move does the first-level gamer; he defines the structures of TBs and gives the decision to down-sided level;

2) the second move does the second-level gamer; he forms the structures of the groups of batches and gives the decision to the third level;.

3) the third-level gamer makes a decision of the groups of batches scheduling, using FTPs; later the decision is given to the second level to analyze the groups of batches optimality;

4) the second-level gamer takes the groups of batches schedules; he allocates the items from to sets and defines the optimality of the batches of such groups. Steps (2-4) are made, while the best decision of the groups structures is not formed; such decision is given to the first level to analyze the optimality of the solutions of batches;

5) the first-level gamer takes the decision of the groups of batches mixture and defines the optimality of the solutions of batches;

6) the upper-level gamer forms a new decision of the the solutions of batches and gives it to a second-level gamer; the solutions of the structure of batches are optimized in consideration of groups of batches, which are taken from the second level.

The gamers' moves are done while the complex decisions, nearly optimal, are achieved. The model of process of proceeding TB in FAL uses the next keys (see Table 1): 
Table 1. The legend of parameters of proceeding TB in FAL

\begin{tabular}{|c|c|}
\hline Legend & The parameter purpose \\
\hline 1 & 2 \\
\hline$i$ & The item's type ID $(i=\overline{1, n})$. \\
\hline$n^{i}$ & The number of type $i$ items. \\
\hline $\mathrm{L}$ & The number of FTP $(l=\overline{l, L})$. \\
\hline$m_{i}$ & The number of Transfer Batch of type i items $(i=\overline{1, n})$. \\
\hline$M$ & The vector of number of TB types i items $(i=\overline{1, n})$. \\
\hline$a_{i h}$ & The number of items $i$ type in $h \mathrm{~TB}\left(h=\overline{1, m_{i}}\right)$. \\
\hline$A$ & Array of mix of items in TB. \\
\hline$\pi^{l}$ & Proceeding sequence of TB in 1 FTP. \\
\hline$P$ & Array of order of sequence of proceeding items of TB in FTP in FAL. \\
\hline$R$ & Array of number of type $i$ items in TB in $\pi^{l}$ obtaining positions $j$. \\
\hline$n_{p}$ & Number of TB items in sequences $\pi^{l}$. \\
\hline$n_{j}$ & Number of items in TB in position $j$ in $\pi^{l}$. \\
\hline$t_{l i}$ & Time of proceeding type $i$ item in FTP $l$. \\
\hline$T$ & Array of times of proceeding of items on FTP in FAL. \\
\hline$t_{i j}^{l}$ & Time of rearrangement $l$ FTP from type $i$-items to type $j$-items proceeding. \\
\hline$T^{l}$ & Arrays of rearrangement of $l$ FTP from type to type of items proceeding. \\
\hline$t_{j q}^{0 l}$ & $\begin{array}{l}\text { The times of the beginning of proceeding } q \text { items in TB, taking } j \text { positions in } \\
\text { proceeding sequence in FAL }\end{array}$ \\
\hline$T^{0 l}$ & $\begin{array}{l}\text { The arrays of times of the beginning of proceeding items in TB, taking } j \\
\text { positions in sequences of proceeding them in } l \text { FTP. }\end{array}$ \\
\hline$t_{j n_{j}}^{O l}$ & $\begin{array}{l}\text { The times of the beginning of proceeding in FAL the last items in TB taking in } \\
\pi^{l} \text { the } \mathrm{j} \text { positions. }\end{array}$ \\
\hline
\end{tabular}

The mathematical model of proceeding TB in FAL considers the definition of values $t_{j q}^{O l}$ for each item, includes in TB and takes a $j$ position in $\pi^{l}$. The recognized model is defined in [1], where the optimization criterion of schedule proceeding of TB, formed on the 3 -rd level of hierarchy, is obtained. The values of $t_{j q}^{0 l}$ are defined using matrices $P$ and $R$. These matrices contain the solve of forming the schedule of proceeding TB in FAL task, when current step of optimizing algorithm is doing.

To give a type of groups of TBs of items, proceeding in FAL, the next keys are used (see Table 2): 
Table 2. The definitions of parameters, using while decision of group mix ot TB items is formed

\begin{tabular}{|c|l|}
\hline Legend & \multicolumn{1}{c|}{ The parameter purpose } \\
\hline$t^{z}$ & ID of time interval of FAL functioning while TB is implemented. \\
\hline$N^{z}$ & The ID of group of TB proceeding during time interval $t^{z}(z=\overline{1, Z})$. \\
\hline$m_{i}^{z}$ & The number of TB type $i$ items, included in group $N^{z}(z=\overline{1, Z})$. \\
\hline$(A)_{i}^{z}$ & The vector of structures of TB type $i$, included in group $N^{z}$. \\
\hline $\mathrm{Q}$ & The set of TB of items, not included in groups $N^{z}(z=\overline{1, Z})$. \\
\hline$m_{i}^{q}$ & The number of of items type $i$, not included in groups $N^{z}$. \\
\hline$(A)_{i}^{q}$ & The vector of structures of TB type $i$ items, not included in groups $N^{z}$. \\
\hline$a_{i}^{z}$ & $\begin{array}{l}\text { The general number of type i items }(i=\overline{1, n}), \text { proceeded in TB in structures } \\
\text { of all groups } N^{z}(z=\overline{1, Z}) .\end{array}$ \\
\hline
\end{tabular}

Using formed keys the decisions on following hierarchy levels of planning systems are:

- first level decision of structures of TB items - $[M, A]$;

- the decision of structures of groups of TB $N^{z}$, proceeding during time intervals $t^{Z}$ $\left.(z=\overline{1, Z}):\left[\left\{N^{Z} \mid(z=\overline{1, Z})\right\}, Q\right]\right)$, where $\left\{N^{Z} \mid z=\overline{1, Z}\right\}$ - the set of sequence of parameters $N^{z}=\left\{\left[i, m_{i}^{z},(A)_{i}^{z}\right]_{k}, \mid k=\overline{1, k_{z}}\right\}^{z}, Q-$ sequence $Q=\left\{\left[i, m_{i}^{q},(A)_{i}^{q}\right]_{k}, \mid k=\overline{1, k_{q}}\right\}$;

- the decision of the processing order of TB in FTM in FAL $\left[P, R,\left\{T^{0 l} \mid l=\overline{1, L}\right\}\right]$.

To formalize the model of proceeding TB of items on FTM in FAL while sets are forming are used the additional keys in Table 3. Recognizing the parameters $n_{g}^{\text {set }}$ and $w_{i g}$ the values of $n^{i}(i=\overline{1, n})$ are evaluating as [8]: $n^{i}=\sum_{g=1}^{g^{\text {set }}} n_{g}^{\text {set }} \cdot w_{i g}$.

Table 3. The definitions, used to build the model of proceeding the TB of items in FTP in FAL, while sets are forming

\begin{tabular}{|c|l|}
\hline Legend & \multicolumn{1}{|c|}{ The parameter purpose } \\
\hline$g$ & ID of the item set. \\
\hline$g^{\text {set }}$ & The general number of type of sets. \\
\hline$n_{g}^{\text {set }}$ & Given number of sets type $g\left(g=\overline{1, g^{\text {set }}}\right)$. \\
\hline$h_{g}$ & Type g set index $\left(h_{g}=\overline{1, n_{g}^{\text {set }}}\right)$. \\
\hline$n_{g}$ & $\begin{array}{l}\text { The number of sets type g, which are to be formed from items, proceeded } \\
\text { in TB in groups } N^{z}(z=\overline{1, Z}) .\end{array}$ \\
\hline$N_{g}$ & $\begin{array}{l}\text { The vector of number of different type of batches, formed from items, } \\
\text { proceeded in TB in groups } N^{z} .\end{array}$ \\
\hline$w_{i g}$ & $\begin{array}{l}\text { The number of items type i, which are in one sets of type g (formed the } \\
\text { component } i \text { of sets of type } g) .\end{array}$ \\
\hline
\end{tabular}

The values $n_{g}$ of different types of sets, formed of items, included in TB, proceeding in groups $N^{Z}$, are estimated using the algorithm of items distribution for sets, similar to 
recognized in [8], while TB of items in groups of items $N^{z}$. As a result of distribution, the values of $n_{g}$ number of sets of types $g$, values $a_{i}^{z}(i=\overline{1, n})$ general number of items type $i$ ( $i=\overline{l, n}$ ), proceeded in groups $N^{z}$, are obtained.

As $\left[\left(a_{h}\right)_{i}^{z}\right]_{k}$ is mentioned the h-th element of vector $(A)_{i}^{z}$, the component of $\mathrm{k}$-th sequence $\left[i, m_{i}^{z},(A)_{i}^{z}\right]_{k}$, using the solution of structure of TB items type $i$, included in group $N^{z}$. The general number of items, proceeded in TB, included in groups $N^{z}$ using the decision $\left[\left\{N^{Z} \mid(z=\overline{1, Z})\right\}, Q, N_{g}\right]$, is valued as $\sum_{z=1}^{Z} \sum_{k=1}^{k_{Z}} \sum_{h=1}^{m_{i}^{Z}}\left[\left(a_{h}\right)_{i}^{Z}\right]_{k}$. The evaluation $\sum_{g=1}^{g} \sum_{i=1}^{\text {set }} n_{g} \cdot w_{i g}$ determines the general number of items, included in sets. The number of items, included in TB in groups $N^{z}(z=\overline{1, Z})$, but not used while sets formed, is evaluated as $\sum_{z=1}^{Z} \sum_{k=1}^{k_{Z}} \sum_{h=1}^{m_{i}^{Z}}\left[\left(a_{h}\right)_{i}^{Z}\right]_{k}-\sum_{g=1}^{g^{s e t}} \sum_{i=1}^{n} n_{g} \cdot w_{i g} . \quad$ The expression $\sum_{g=1}^{g^{s e t}} n_{g}$ determines the number of sets, formed from proceeded items in TB in groups $N^{z}(z=\overline{1, Z})$. The model of hierarchical game to optimize a decision using a structure of TB, groups of TB and schedules of proceeding TB of groups in FAL is:

$$
\text { 1st level: } \max f_{1}\left([M, A], N_{g}\right) \text {, }
$$

where $f_{1}=\sum_{g=1}^{g^{\text {set }}} n_{g}$

2nd level: $\min f_{2}\left([M, A],\left[\left\{N^{z} \mid(z=\overline{1, Z})\right\}, Q, N_{g}\right]\right)$,

where $f_{2}=\sum_{z=1}^{Z} \sum_{k=1}^{k_{Z}} \sum_{h=1}^{m_{i}^{Z}}\left[\left(a_{h}\right)_{i}^{Z}\right]_{k}-\sum_{g=1}^{g^{s e t}} \sum_{i=1}^{n} n_{g} \cdot w_{i g}$;

3rd level $[1,8,9]: \min f_{3}^{z}\left(N^{z},\left\{\left[P^{z}, R^{z},\left\{\left(T^{0 l}\right)^{z} \mid(l=\overline{1, L})\right\}\right]\right)\right.$

$(z=\overline{1, Z})$,

where

$$
\begin{aligned}
f_{3}^{z} & =\sum_{l=2}^{L}\left[t_{l 1}^{0 l}\right]^{z}+\sum_{l=1}^{L} \sum_{j=2}^{n_{p}^{z}}\left[\left[t_{j 1}^{0 l}\right]^{z}-\left[\left[t_{j-l, n_{j-1}}^{0 l}\right]^{z}+\sum_{h=1}^{k_{z}}[t l h]^{z} \cdot p_{h, j-1}^{z}\right]\right]+ \\
& +\sum_{l=2}^{L} \sum_{j=l}^{n_{p}^{z}} \sum_{q=2}^{n_{j}^{z}}\left[\left[t_{j q}^{0 l}\right]^{z}-\left[\left[t_{j, q-1}^{0 l}\right]^{z}+\sum_{h=1}^{k_{z}}\left[t_{l h}\right]^{z} \cdot p_{h j}^{z}\right]\right] ;
\end{aligned}
$$

the time of proceeding limit of TB included in groups $N^{z}(z=\overline{1, Z})$ [9]:

$$
\left[\left(t_{n_{p}^{O L}, n_{n_{p}^{z}}}^{O L}\right)^{z}+\sum_{i=1}^{k_{z}}\left(t_{L i}\right)^{z} \cdot p_{i, n_{p}^{z}}^{z}\right] \leq t^{z} \text { at } z=\overline{1, Z} \text {. }
$$

The 1st level criterion follows the integrated number of sets formed., the 2nd level criterion - the number of sets, proceeded in TB, included in groups, not included to forming sets, 3rd level criterion suits to general times of non-functioning of FTM while TB in group is proceeded. The expression (4) recognizes, that the end of proceeding time of last TB in L-th FTM is not more than time of the end of interval of $t^{z} \quad(z=\overline{1, Z})$ of FAL functioning. The optimization of the structure of TB is done using the method, recognized in [1]. 
The decision $\left[\left\{N^{z} \mid(z=\overline{1, Z})\right\}, Q, N_{g}\right] *$ of mix of groups of TB is obtained by the next steps: 1) the initial decision of structure of groups [9]; 2) the evaluating of decisions in local area optimal decision and choosing the best of them. The algorithm of initial decision of structure of groups forming $N^{z}(z=\overline{l, Z})$ [9] recognizes the sequential distribution of TB to groups using limit (expression (4)). The sets forming is done using the values of general number type i items, signed as $a_{i}^{z}(i=\overline{1, n})$, the batches of which are included in groups $N^{z}$ $(z=\overline{1, Z})$, values using expression $a_{i}^{z}=\sum_{z=1}^{Z} \sum_{h=1}^{m_{i}^{Z}}\left(a_{h}\right)_{i}^{z}(i=\overline{1, n})$. After the algorithm is realized, values $a_{i}^{z}>0$ are described the number of type i items, not included in sets.

The optimization of structures of groups of TB considers the definition for every batch of every type of batches of tasks type $i$, included in group $N^{z}$, the values of difference between of number of tasks $\left(a_{h}\right)_{i}^{z}\left(h=\overline{1, m_{i}^{z}}\right)$ in batch and $a_{i}^{z}$, evaluated after the distribution by sets is done (using $\left(a_{h}\right)_{i}^{z}>a_{i}^{z}$ and $a_{i}^{z}>0$ ). The party with the minimal difference $i$-of number of items $\left(a_{h}\right)_{i}^{z}$, where the values of $a_{i}^{z}$ are the least, is chosen. To eval the deviation (difference) of items of $\left(a_{h}\right)_{i}^{z}\left(h=\overline{1, m_{i}^{z}}\right)$ in parties of type $i$ from $a_{i}^{z}>0$, the parameter $m h_{i}^{z}$, evaluated by expression : $m h_{i}^{z}=\min _{1 \leq h \leq m_{i}^{z}}\left(\left(a_{h}\right)_{i}^{z}-a_{i}^{z}\right)$ (witn $\left.\left(\left(a_{h}\right)_{i}^{z}-a_{i}^{z}\right)>0\right)$ is considered.

The way to optimize the groups of TB is assumed to do the steps:

- the values of $n_{g}$ of formed batches of type $g$ after the proceeded items are distributed, after the number of formed sets and the values of $a_{i}^{z}(i=\overline{1, n})$, that $a_{i}^{z} \geq 0$; if $a_{i}^{z}>0$, there are not distributed to sets items;

- for each type $i$ item, TB of which is included to group $N^{z}$, the value of $a_{i}^{z}>0$ is checked, while true, in group $N^{z}$ the choose of batch of this type of item is done. The number of batch is $h_{i}^{z}$, the difference between items in it from $a_{i}^{z}>0$ is minimal (equals to $m h_{i}^{z}$ while $\left(\left(a_{h}\right)_{i}^{z}-a_{i}^{z}\right) \geq 0$ и $\left.a_{i}^{z}>0\right)$;

- $h_{i}^{z}$-th TB of type $i$ items excludes from group $N^{z}$, instead of it the batch of different types of items are added to group, not included in groups $N^{z}(z=\overline{1, Z})$ (from $Q$ ); the added batch should provide the maximal reduced by value $f_{2}(\cdot)$ decision;

- the steps to exclude batch from group $N^{z}$ and addition batches from the $Q$, are done for every types if items with $a_{i}^{z}>0$; to consider group the parameters of excluding and including bathes are recorded to allow to form a decision with reducing the value of $f_{2}(\cdot)$ criterion to maximal degree;

- the steps to exclude batches type $i(i=\overline{1, n})$ and including the batches from $Q$, are done for each group $N^{z} \quad(z=\overline{1, Z})$; among the decisions of structures of groups $N^{z}$ $(z=\overline{1, Z})$ the local optimal decision is the one, to which the reducing of $f_{2}(\cdot)$ value is maximal. 
The considered way of optimization of decision by structures of TB groups is implemented as a program. The researches of the specific characteristics of scheduling of proceeding TB in FAL while sets are formed and times of line functioning is done are made.

The goal of the research of planning of TB is the definition of number of sets using the local optimization decision by mix of groups to compare with a fixed groups of TB (the structures of groups are the initial decisions). The function of the ratio of $f_{\text {mosg }}=\left(f_{1}^{o s g b}-f_{1}^{f i x}\right) / f_{1}^{f i x}$ from the parameters of task is considered as a result $\left(f_{\text {mosg }}-\right.$ the measure of increasing of sets number while the structures of groups of TB are optimized, $f_{l}^{f i x}$ - the criterion $f_{l}(\cdot)$ value, if the structures of groups are not optimized (fixed groups of TB), $f_{l}^{o s g b}$ - the value of the criterion $f_{l}(\cdot)$ for optimizing using the considered method). The researches of schedule planning of processing of TB in FAL are done with using the next input parameters: $\max \left(t_{i}^{l}\right) / \min \left(t_{i}^{l}\right)$ - the ration of the maximal time of proceeding of items to minimal time of proceeding items in $\mathrm{FTM} ; \max \left(t_{i j}^{l}\right) / \min \left(t_{i k}^{l}\right)$ - the ratio of maximal time of rearrangement of FTM in FAL to minimal rearrangement time of FTM; $\max \left(w_{i g}\right) / \min \left(w_{i g^{\prime}}\right)$ - the ration of maximal number of items type $i$ while sets type $g$ is formed, to minimal number of items type $i$ while sets type $g$ is formed. The values $\max \left(t_{i j}^{l}\right) / \min \left(t_{i k}^{l}\right)$ were done equal to $1,2,4,8$; the values $\max \left(t_{i}^{l}\right) / \min \left(t_{i}^{l}\right)$ - were done equal to $1,2,4$; the values of $\max \left(w_{i g}\right) / \min \left(w_{i g^{\prime}}\right)$ - were done equal to $1,2,4$. The values of parameter $n_{g}^{\text {set }}$, were equaled to 4 and 8 . The value of $g^{\text {set }}$ equaled to 4 . While $n_{g}^{\text {set }}=4$ time intervals $t^{z}(z=\overline{1,3})$ were equaled to 100 , while $n_{g}^{\text {set }}=8$ time intervals $t^{z}$ $(z=\overline{1,3}$ ) were equaled 200. Investigating the efficiency of planning of proceeding TB in FAL the decisions were formed: fixed groups of TB; optimized by structures of groups of TB using the considered method. As a result, the next values were obtained: $n_{g}^{\text {set }}=4, t^{z}=100, \mathrm{Z}=3$, $\max \left(w_{i g}\right) / \min \left(w_{i g^{\prime}}\right)=1$ the maximal value of $f_{\text {mosg }}$ equals to 0.65 (increasing a number of formed sets up to $65 \%)$. If $n_{g}^{\text {set }}=4, t^{z}=100, \mathrm{Z}=3, \quad \max \left(w_{i g}\right) / \min \left(w_{i g^{\prime}}\right)=2$ maximal value of $f_{\text {mosg }}$ equals to $0.75 ;$ if $n_{g}^{\text {set }}=8, t^{z}=200, \quad \mathrm{Z}=3$, $\max \left(w_{i g}\right) / \min \left(w_{i g^{\prime}}\right)=1$ maximal value of $f_{\text {mosg }}$ equals to 0.8 ; if $n_{g}^{\text {set }}=8, t^{z}=200$, $\mathrm{Z}=3, \max \left(w_{i g}\right) / \min \left(w_{i g^{\prime}}\right)=2$ maximal value of $f_{\text {mosg }}$ equals to 0.85 . The computational complexity of algorithm of optimizing the groups of TB is defined as $O\left(n \cdot n^{i} \cdot Z\right)$.

\section{Conclusion}

The modern development of approaches of complex scheduling of proceeding of TB in FAL is described by lack of optimizing methods to optimize the decision using structures and schedule of processes them in FTM, when the limits to size of task are not considered, and the methods with assurance of coming close to optimal decision, while different values of input 
data are used. The methods of defining the structures of TB are not considered the specifics of task, using a necessity of forming the sets with proceeded in FAL items and time limits of FAL functioning. The hierarchical approach means the definition of the structures of batches, structures of groups of batches and scheduling in FAL, using the hierarchical ordered levels of the planning system (hierarchical games theory). The model of hierarchical game while optimizing the structures of bathes, groups of TB and schedule of proceeding them in FAL are developed. The results showed the ability of significant increasing of forming sets from items, proceeded in TB in groups.

\section{References}

1. Krotov K.V. A complex method for determining effective solutions for the composition of data batches and schedules of their processing in conveyor systems.// Computational technologies, Publishing house Of the Institute of computational technologies SB RAS, Vol. 23, \# 3, 2018. pp. 58-76 (In Russ).

2. Mendez C.A. et al. State-of-the-art review of optimization methods for short-term scheduling of batch processes.//Computers and Chemical Engineering, 2006, vol. 30. P. 913-946.

3. Agha M. Integrated management of energy and production: scheduling of batch process and Combined Heat\& Power plant. // University of Toulouse, National Polytechnic Institute of Toulouse (FRANCE), 2009, 255 p.

4. Kovalev M.M. Models and methods of calendar planning: a course of lectures.- Minsk: Publishing house of BSU. 2004. 63 p. (In Russ).

5. Chandra P. Managing Batch Processors to reduce lead time in a semiconductor packaging line.// International Jornal of Production Research, 1997, vol. 35(3). P. 611-633.

6. Steiner G., Zhang R. Minimizing the weight number of late jobs with Batch setup times and delivery costs on a single machine.//Vienna: Itech Education and Published, 2007, 436 p.

7. Koehler F. Optimal Batch Schedules for Parrallel Machines.// Algorithms and Data Structures: 13th International Symposium. Berlin: Springer, 2013. P. 475-486.

8. Krotov K. V. Justification of methods for constructing complex schedules for processing data batches under the condition of rapid formation of sets of results.// Bulletin Of The Voronezh State University. Series: System analysis and information technologies. \#4, 2018, pp. 58-72 (In Russ).

9. Krotov K. V. Construction of complex schedules for processing data packets in a pipeline system when setting restrictions on the duration of time intervals of its operation.// Proceedings of educational institutions of communication, 2020, vol. 6, No. 3. pp. 75-89 (In Russ). 